\title{
Association Between Admission Serum Phosphate Level and All-Cause Mortality Among Patients with Spontaneous Intracerebral Hemorrhage
}

\author{
Yu Hong ${ }^{1,2, *}$ \\ Xian-Hui Wang ${ }^{3, *}$ \\ Yi-Tong Xiong ${ }^{2}$ \\ Jie $\mathrm{Li}^{2}$ \\ Chun-Feng Liu (D) \\ 'Department of Neurology, The Second \\ Affiliated Hospital of Soochow University, \\ Suzhou, Jiangsu, 2I5004, People's \\ Republic of China; ${ }^{2}$ Department of \\ Neurology, Affiliated Yixing People's \\ Hospital of Jiangsu University, Yixing, \\ jiangsu, 214200, People's Republic of \\ China; ${ }^{3}$ Department of Neurology, \\ Taicang First People's Hospital, Taicang, \\ Jiangsu, 215400, People's Republic of \\ China
}

*These authors contributed equally to this work
Correspondence: Jie Li

Department of Neurology, Yixing People's Hospital, No. 75 Tongzhenguan Road, Yicheng Street, Yixing, Jiangsu, 214200, People's Republic of China Email jieleel983@I63.com

Chun-Feng Liu

Department of Neurology, The Second Affiliated Hospital of Soochow University, No. 1055 Sanxiang Road, Suzhou, Jiangsu, 215004, People's Republic of China

Email liuchunfeng@suda.edu.cn
Background: Hypophosphatemia was reported to frequently occur in patients with nontraumatic intracranial hemorrhage (ICH); however, the correlation between hypophosphatemia and outcomes of ICH remains unclear. This study aimed to examine the association between admission serum phosphate and all-cause mortality among patients with mildmoderate spontaneous ICH (sICH).

Methods: A total of 851 patients with sICH were enrolled. Serum phosphate was acquired within 24 hours on admission, and participants were divided according to phosphate quartiles. The primary outcome was all-cause mortality within 90 days, and univariate and multivariate models were employed to estimate the mortality risk.

Results: There were significant differences among sICH patients with different phosphate quartiles in terms of age, diastolic blood pressure (DBP), activated partial thromboplastin time (APTT), platelet count, and incidence of respiratory failure events on admission $(P<0.05)$. Log rank test showed a significant difference in the mortality risk among sICH patients with each phosphate quartile. Univariate Cox regression analysis revealed that age, smoking, DBP, APTT, NIH stroke scale (NIHSS) score, hematoma volume and serum phosphate might be associated with the 90-day all-cause mortality in patients with $\operatorname{siCH}(P<0.05)$. Multivariable Cox regression analysis showed that the crude mortality was 4.3 -fold greater in sICH patients with serum phosphate Q1 than those with Q4 $(P<0.001)$, and remained 3.18-fold higher after adjusting for age, smoking, DBP, APTT, NIHSS score, hematoma volume and early withdrawal of life-sustaining therapy $(P=0.011)$. Representative operating curve (ROC) analysis showed that admission serum phosphate was predictable for all-cause mortality within 90 days in patients with $\mathrm{sICH}$ (area under the $\mathrm{ROC}=0.628, P<0.001$ ).

Conclusion: Low admission serum phosphate is strongly associated with a high risk of mortality in patients with mild-moderate $\mathrm{sICH}$, and hypophosphatemia may be a prognostic marker for all-cause mortality in patients with mild-moderate sICH.

Keywords: spontaneous intracerebral hemorrhage, serum phosphate level, risk factor, allcause mortality

\section{Introduction}

Currently, spontaneous intracerebral hemorrhage (sICH) remains a global public health concern. ${ }^{1}$ As the second most frequent type of stroke, this devastating and deadly neurological emergency is associated with a significantly high global burden of disease. ${ }^{2}$ It is estimated that sICH has an annual incidence of approximately $25 /$ 100,000 worldwide, and is characterized by a high mortality of approximately $40 \%$ 
at 1 month and more than $60 \%$ at 1 year. ${ }^{3}$ The etiology of sICH is considered heterogeneous and complex, and systemic arterial hypertension, excess alcohol consumption, male, advanced age, and smoking history have been identified as the major risk factors for sICH. ${ }^{4}$ Since there is no effective treatment for this disorder until now, ${ }^{5-7}$ early identification and timely interventions are accepted to be critical for improving the clinical outcomes in patients with sICH. ${ }^{8}$ Nevertheless, early and precision prediction of the prognosis remains a great challenge among patients with sICH. ${ }^{9}$

Serum phosphate is an important element in the maintenance of normal cell functions. ${ }^{10}$ Phosphate presents in various forms in the human body and contributes an important role to life activities. ${ }^{11}$ As a common condition in critically ill patients, ${ }^{12}$ hypophosphatemia has been found to be associated with multiple clinical symptoms, including cardiovascular events, respiratory failure and hematological disease. $^{13-15}$ Low phosphate level was reported to correlate with an increased risk of brain infarction in hemodialysis patients, ${ }^{16}$ and results from a prospective, observational study showed a $70 \%$ detection rate of hypophosphatemia among patients with nontraumatic ICH. ${ }^{17}$ In addition, no significant association was found between serum phosphate and excellent outcomes among patients with acute $\mathrm{ICH}{ }^{18}$ However, the correlation between serum phosphate and the clinical outcome has not been fully demonstrated in patients with sICH. Based on previous reports, it is hypothesized that hypophosphatemia may be associated with the risk of mortality among ICH patients. To test our hypothesis, this retrospective study was designed with aims to examine the association between admission serum phosphate level and all-cause mortality among patients with mild-moderate sICH.

\section{Materials and Methods}

\section{Ethical Statement}

The study was approved by the Ethics Review Committee of Yixing People's Hospital (approval number: IRB-2019-ARTICLE-001). All procedures were performed in accordance with the Declaration of Helsinki and international and national guidelines for human studies. Written informed consent was obtained from all participants following a detailed description of the purpose of the study.

\section{Subjects}

A total of 891 patients with sICH that were hospitalized in Yixing people's Hospital (Yixing, China) during the period from January 2015 to April 2020 were recruited. The inclusion criteria were assigned as follows: (1) detection of sICH with computerized tomography (CT) scan within 24 hours from disease onset; (2) serum phosphate available on admission; and (3) available demographic data, laboratory data, imaging data, medical history, treatment history and clinical scores. Patients with the following criteria were excluded from the study: (1) traumatic $\mathrm{ICH}, \mathrm{ICH}$ due to intracranial tumor or vascular malformations, hemorrhagic conversion of acute ischemic stroke; (2) presence of massive cerebral hemorrhage that required neurosurgical procedures; or (3) chronic kidney disease (stage 3-5), kidney dysfunction (serum creatinine $>$ $200 \mathrm{~mol} / \mathrm{L}$ ). Mortality data were confirmed by followup phone communications. Among the enrolled 891 participants, 40 subjects were excluded because of incomplete baseline data (13 subjects), requirement of surgical treatment due to disease aggravation (16 subjects), and unavailable data (11 subjects), and finally, 851 patients were included in our study.

\section{Imaging Analysis}

All images were evaluated by two neuroradiologists blinded to demographic and clinical features. Baseline hematoma volumes were assesses using the $A B C / 2$ formula based on CT scans, where $A$ is the greatest hemorrhage diameter by CT scan, $B$ is the diameter $90^{\circ}$ to $A$, and $C$ is the approximate number of $\mathrm{CT}$ slices with hemorrhage multiplied by the slice thickness. ${ }^{19}$ Cranial CT scan was routinely performed on admission, at 24-hour after admission or in cases of disease aggravation.

\section{Data Collection}

Patients' demographic characteristics (age, gender), lifestyle risk factors (smoking, drinking), history of chronic diseases and all complications during the hospital stay (including cardiovascular events, respiratory failure and hematological disease) were captured from 
medical records. Systolic blood pressure (SBP) and diastolic blood pressure (DBP) were routinely measured in a supine position. Levels of serological markers were routinely measured on admission, including triglyceride (TG), low-density lipoprotein cholesterol (LDL-C), high-density lipoprotein cholesterol (HDLC), activated partial thromboplastin time (APTT), platelet, creatinine and serum phosphate level. All subjects were classified into four groups according to the quartile of serum phosphate levels $(\mathrm{Q} 1,<0.9 \mathrm{mmol} / \mathrm{L}$; Q2, 0.9-1.01 mmol/L; Q3, 1.02-1.14 mmol/L; Q4, > $1.14 \mathrm{mmol} / \mathrm{L}$ ). Neurological impairment was assessed by National Institutes of Health Stroke Scale (NIHSS) on admission, ${ }^{20}$ and the time of death as confirmed by medical records and phone communications with family members.

\section{Statistics}

All normally distributed measurement data were presented as mean \pm standard deviation (SD), and nonnormal distributed variables were presented as median (interquartile spacing), while categorical parameters were expressed as numbers (percentages). One-way analysis of variance (ANOVA) was employed to compare the baseline characteristics on admission among the four groups followed by post-hoc Bonferroni's multiple comparison tests, and differences of proportions were tested for statistical significance with chisquare test. The cumulative incidence was estimated using the Kaplan-Meier method, and Log rank test was used for comparisons among groups. Cox proportional hazards regression analysis was used to estimate hazard ratio $(H R)$ and its $95 \%$ confidential interval $(C I)$. The confounding factors, including age, smoking, DBP, APTT, NIHSS score on admission, hematoma volume and early withdrawal of life-sustaining therapy, were adjusted in different multivariate Cox regression models. The performance of admission serum phosphate level for predicting the mortality risk was evaluated using receiver operating characteristic curve (ROC) analysis among patients with sICH. All statistical analyses were performed using the statistical package SPSS version 23.0 (SPSS, Inc.; Chicago, IL, USA), and a $P$ value of $<0.05$ was considered statistically significant.

\section{Results}

\section{Subject Characteristics}

The 851 participants included 563 males and 288 females, and had a mean age of $65.3 \pm 13.5$ years. There were 28 subjects (3.3\%) dying from all causes within 30 days, and 80 cases $(9.4 \%)$ dying from all causes within 90 days. There were significant differences among sICH patients with four phosphate quartiles in terms of age, DBP, APTT, platelet count, and incidence of respiratory failure events on admission $(P<0.05)$ (Table 1$)$.

\section{Association of Admission Serum Phosphate with the Risk of Mortality in sICH Patients}

The Kaplan-Meier method was employed to estimate the cumulative risk of mortality, and Log rank test showed a significant difference in the risk of mortality among sICH patients with each serum phosphate quartile (Figure 1). Univariate Cox regression analysis revealed that age, smoking, DBP, APTT, NIHSS score, hematoma volume and serum phosphate were associated with the 90-day all-cause mortality among patients with $\operatorname{sICH}(P<0.05)$ (Table 2). Multivariable Cox regression analysis showed that the crude mortality was 4.3 -fold $(95 \% \mathrm{CI}: 1.98-9.33)$ greater in $\mathrm{sICH}$ patients with serum phosphate Q1 than those with Q4 $(P<0.001)$, and remained 3.18-fold higher $(95 \% C I$ : 1.57-7.82) after adjusting for age, smoking, DBP, APTT, NIHSS score, hematoma volume and early withdrawal of life-sustaining therapy $(P=0.011)$ (Table 3$)$. ROC analysis showed that admission serum phosphate level was predictable for all-cause mortality within 90 days in patients with sICH [area under ROC (AUC) = 0.628, $P<0.001$ ) (Figure 2), and the joint use of admission serum phosphate, hematoma expansion, presence of intraventricular hemorrhage (IVH) and ICH volume on admission increased the predictive value $(\mathrm{AUC}=0.785, P<0.001)$ (Figure 3$).$ Our data indicate that admission serum phosphate level may be a prognostic factor for all-cause mortality within 90 days in patients with mild-moderate $\mathrm{sICH}$.

\section{Discussion}

Hypophosphatemia, a common phenomenon in patients with nontraumatic $\mathrm{ICH},{ }^{17}$ is also observed in individuals 
Table I Demographic and Clinical Characteristics on Admission Among Patients with sICH with Different Serum Phosphate Quartiles

\begin{tabular}{|c|c|c|c|c|c|}
\hline \multirow[t]{2}{*}{ Characteristics } & \multicolumn{4}{|c|}{ Serum Phosphate (mmol/L) } & \multirow[t]{2}{*}{$P$ value } \\
\hline & QI $(<0.90)$ & Q2(0.90-I.0I) & Q3(1.02-1.14) & Q4(> I.14) & \\
\hline $\mathrm{N}$ & 207 & 213 & 225 & 206 & \\
\hline Age (years) & $68.18 \pm 12.80$ & $64.23 \pm|2.5|$ & $65.20 \pm 13.77$ & $62.49 \pm 14.10$ & $<0.001$ \\
\hline Male, n (\%) & $150(72.5)$ & $150(70.4)$ & $140(62.2)$ & $123(59.7)$ & 0.103 \\
\hline Smoking, n (\%) & $29(14.0)$ & $39(18.3)$ & $28(12.4)$ & $30(14.6)$ & 0.330 \\
\hline Drinking, n (\%) & $23(11.1)$ & $23(10.8)$ & $16(7.1)$ & $28(13.6)$ & 0.217 \\
\hline History of hypertension, $\mathrm{n}$ (\%) & $169(81.6)$ & $178(83.6)$ & $178(79.1)$ & I $78(86.4)$ & 0.296 \\
\hline History of diabetes, n (\%) & $39(18.8)$ & $45(21.1)$ & $42(18.7)$ & $36(17.5)$ & 0.678 \\
\hline SBP $(\mathrm{mmHg})$ & $165.61 \pm 25.47$ & $166.00 \pm 27.36$ & $|66.70 \pm 28.5|$ & $|66.72 \pm 26.7|$ & 0.614 \\
\hline $\mathrm{DBP}(\mathrm{mmHg})$ & $90.72 \pm 14.38$ & $93.64 \pm 15.52$ & $94.28 \pm 17.13$ & $95.82 \pm 16.66$ & 0.013 \\
\hline TG (mmol/L) & $1.35 \pm 1.26$ & $1.42 \pm 1.31$ & $1.50 \pm 1.29$ & $1.47 \pm 1.33$ & 0.617 \\
\hline LDL-C (mmol/L) & $2.53 \pm 0.65$ & $2.63 \pm 0.77$ & $2.70 \pm 0.72$ & $2.74 \pm 0.82$ & 0.577 \\
\hline HDL-C (mmol/L) & $1.18 \pm 0.26$ & $1.21 \pm 0.30$ & $1.19 \pm 0.30$ & $1.18 \pm 0.33$ & 0.502 \\
\hline APTT (s) & $31.75 \pm 6.21$ & $30.06 \pm 4.00$ & $29.88 \pm 4.47$ & $30.14 \pm 4.96$ & 0.003 \\
\hline Platelet $\left(10^{9} / \mathrm{L}\right)$ & $|84.64 \pm 60.2|$ & $190.76 \pm 64.58$ & $190.39 \pm 62.00$ & $204.42 \pm 67.85$ & 0.009 \\
\hline Creatinine $(\mu \mathrm{mol} / \mathrm{L})$ & $59.14 \pm 26.97$ & $60.55 \pm 41.63$ & $58.85 \pm 36.46$ & $65.82 \pm 50.78$ & 0.314 \\
\hline Time from onset $(h)$ & $3(2-13)$ & $3(2-16)$ & $3(2-24)$ & $3(2-17)$ & 0.702 \\
\hline Baseline NIHSS score & $6(2-11)$ & $7(3-11)$ & $6(2-11)$ & $5(2-10)$ & 0.459 \\
\hline Hematoma volume $(\mathrm{mL})$ & $10.2(4.8-18.0)$ & II.6 (5.7-16.0) & $10.0(4.2-16.6)$ & II.3 (5.5-16.5) & 0.416 \\
\hline \multicolumn{6}{|l|}{ Hematoma location, n (\%) } \\
\hline Intraventricular hemorrhage & $48(23.2)$ & $43(20.2)$ & $49(21.8)$ & $43(20.8)$ & 0.891 \\
\hline Lobe & $32(15.5)$ & $25(11.7)$ & $35(15.6)$ & $29(14.1)$ & 0.644 \\
\hline Basal ganglia & $112(54.1)$ & $136(63.8)$ & $123(54.7)$ & $|2|(58.7)$ & 0.149 \\
\hline Cerebellum & $10(4.8)$ & $5(2.3)$ & $7(3.1)$ & $8(3.9)$ & 0.562 \\
\hline Brain stem & $9(4.3)$ & $4(1.9)$ & $6(2.7)$ & $6(2.9)$ & 0.505 \\
\hline \multicolumn{6}{|l|}{ Event outcomes } \\
\hline Cardiovascular event & $22(10.6 \%)$ & $16(7.5 \%)$ & $20(8.9 \%)$ & II (5.4\%) & 0.249 \\
\hline Respiratory failure event & 32 (15.5\%) & 16 (7.5\%) & 21 (9.3\%) & 19 (9.2\%) & 0.042 \\
\hline Hematoma expansion & $45(21.7 \%)$ & 39 (I8.3\%) & 43 (19.1\%) & $38(18.4 \%)$ & 0.462 \\
\hline
\end{tabular}

after severe traumatic brain injury; ${ }^{21-24}$ however, there is little knowledge on the correlation between hypophosphatemia and prognosis in patients with sICH. Our data demonstrate that low admission serum phosphate may be an independent risk factor of all-cause mortality within 90 days in patients with mild-moderate sICH.

Hypophosphatemia has been found to occur after neurological disorders. ${ }^{25} \mathrm{~A}$ recent study reported that the 


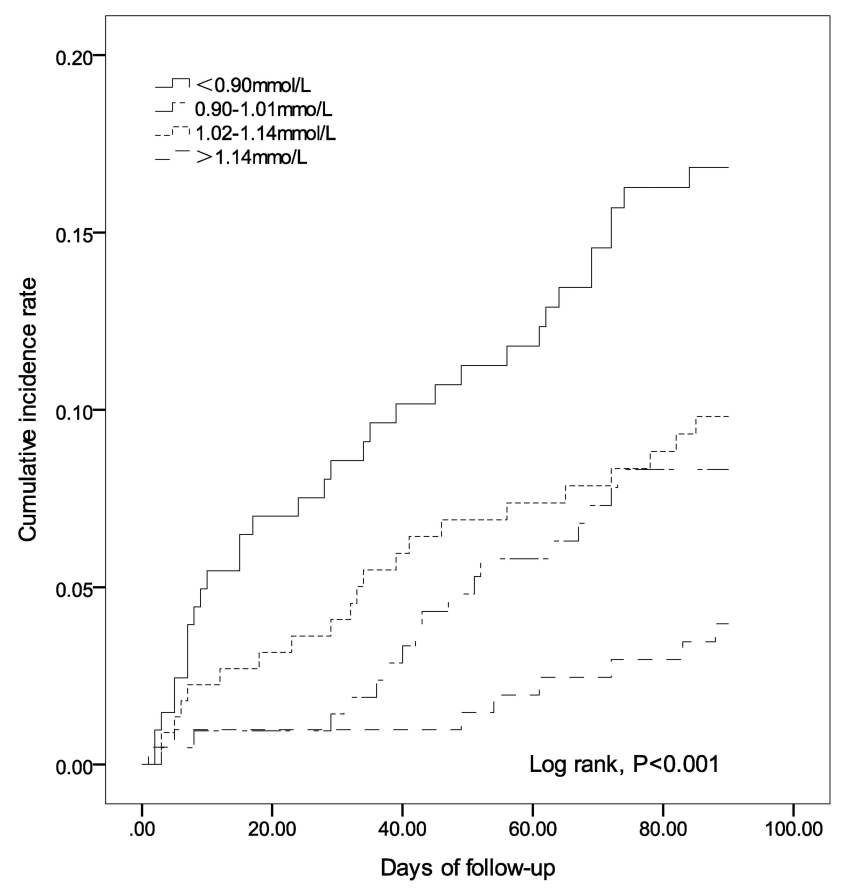

Figure I Kaplan-Meier curve estimates the mortality in spontaneous intracerebral hemorrhage patients with different admission serum phosphate quartiles. Log rank test revealed significant differences in the cumulative mortality among spontaneous intracerebral hemorrhage patients with different admission serum phosphate quartiles $(P<0.001)$

glucose-phosphate ratio was a potential marker for 3-month satisfactory outcomes in 198 patients with aneurysmal subarachnoid hemorrhage. ${ }^{26}$ Similarly, serum

Table 2 Univariate Cox Regression Analysis for 90-Day AllCause Mortality Among Patients with sICH

\begin{tabular}{|l|c|c|c|}
\hline Variables & $\boldsymbol{\beta}$ & HR (95\% CI) & $\boldsymbol{P}$ value \\
\hline Age (years) & 0.093 & $1.097(1.073-1.122)$ & $<0.001$ \\
\hline Male sex & 0.204 & $0.815(0.501-1.326)$ & 0.411 \\
\hline Smoking & 1.521 & $0.218(0.069-0.693)$ & 0.010 \\
\hline History of hypertension & 0.440 & $0.044(0384-1.080)$ & 0.095 \\
\hline History of diabetes & 0.454 & $1.574(0.954-2.596)$ & 0.076 \\
\hline SBP & 0.006 & $1.006(0.998-1.014)$ & 0.131 \\
\hline DBP & -0.017 & $0.983(0.968-0.999)$ & 0.034 \\
\hline LDL-C & -0.205 & $0.814(0.589-1.127)$ & 0.216 \\
\hline APTT & -0.065 & $0.937(0.889-0.987)$ & 0.014 \\
\hline Platelet & 0.000 & $1.000(0.996-1.003)$ & 0.967 \\
\hline NIHSS score & 0.107 & $1.113(1.093-1.133)$ & $<0.001$ \\
\hline Hematoma volume & 0.168 & $1.186(1.150-1.223)$ & $<0.001$ \\
\hline Serum phosphate level & -0.397 & $0.215(0.064-0.719)$ & 0.013 \\
\hline
\end{tabular}

glucose-phosphate ratio was found to correlate with severity and 6-month mortality in patients with severe traumatic brain injury. ${ }^{27}$ Conversely, You and colleagues ${ }^{18}$ reported no significant association between serum phosphate and excellent outcomes among patients with acute ICH. The diverse correlation between serum phosphate and prognosis of patients with ICH may be attributed to the different subjects' characteristics and study designs.

In the current study, we found that lower serum phosphate levels were associated with a higher risk of 90-day all-cause mortality in patients with mild-moderate $\mathrm{sICH}$, and it is hypothesized that serum phosphate level may be a prognostic factor for mild-moderate sICH. First, phosphate is an important component of cell membranes and plays a significant role in mediating intracellular signaling, and therefore, low serum phosphate may affect vascular biology. ${ }^{28,29}$ Second, phosphate is an important component in producing adenosine tri phosphate (ATP) and 2,3-diphosphoglycerate, which mainly promotes the release of oxygen from hemoglobin. ${ }^{30,31}$ Phosphate repletion causes the dysfunction of ATP degradation and induces higher energy charge in mitochondria, ${ }^{31}$ and hypophosphatemia has been found to increase the affinity of hemoglobin, leading to reduced oxygen release. ${ }^{32}$ Therefore, phosphate repletion may impair the brain energy metabolism and result in brain injury, ${ }^{32}$ while phosphate supplementation could reverse the clinical symptoms of neurological diseases and disturbances. ${ }^{33}$ These data strongly support our findings. Finally, a lower LDL-C level was found to cause an increased risk of morality after $\mathrm{ICH}^{34}$ In this study, we found that the sICH patients with serum phosphate Q1 had the lowest LDL-C level, which may be an indirect explanation for the significant difference in the all-cause mortality. However, the exact mechanisms underlying the correlation between lower serum phosphate and a higher risk of mortality among patients with $\mathrm{sICH}$ require further large-scale prospective clinical trials.

Our study has several limitations. First, in this single-center, retrospective study, most patients with $\mathrm{sICH}$ did not receive the measurement of parathyroid functions, vitamin D blood levels or blood gas analysis during the hospital stay, and these patients cannot be excluded from the study. Therefore, the selection bias cannot be completely eliminated, which may affect the 
Table 3 Multivariable Cox Regression Analysis for 90-Day All-Cause Mortality Among sICH Patients with Different Serum Phosphate Quartiles

\begin{tabular}{|c|c|c|c|c|c|}
\hline \multirow[t]{2}{*}{ Mortality Risk } & \multicolumn{4}{|c|}{ Serum Phosphate (mmol/L) } & \multirow[t]{2}{*}{$P$ value } \\
\hline & QI & Q2 & Q3 & Q4 & \\
\hline No. of deaths (\%) & $33(15.9)$ & $17(8.0)$ & $22(9.8)$ & $8(3.9)$ & \\
\hline Crude mortality $(\%, 95 \% \mathrm{Cl})$ & $4.3(1.98-9.33)$ & $2.10(0.91-4.86)$ & $2.49(1.10-5.63)$ & I & $<0.001$ \\
\hline Mortality using Model I $(\%, 95 \%$ Cl) & $2.7(1.23-5.91)$ & $1.82(0.78-4.24)$ & $1.78(0.78-4.06)$ & I & 0.011 \\
\hline Mortality using Model $2(\%, 95 \% \mathrm{Cl})$ & $3.25(1.47-7.26)$ & $1.57(0.57-3.67)$ & $1.59(0.69-3.67)$ & 1 & 0.001 \\
\hline Mortality using Model $3(\%, 95 \% \mathrm{Cl})$ & $3.51(1.57-7.82)$ & $2.31(0.98-5.44)$ & $1.95(0.84-4.55)$ & I & 0.001 \\
\hline Mortality using Model 4 (\%, 95\% Cl) & $3.18(1.48-6.7)$ & I.69 (0.73-3.93) & $1.74(0.76-4.00$ & I & 0.011 \\
\hline
\end{tabular}

Notes: Model I: adjusted for age, smoking, DBP and APTT; Model 2: adjusted for age, smoking, DBP, APTT and baseline NIHSS score; Model 3: adjusted for age, smoking, DBP, APTT, baseline NIHSS score and hematoma volume; Model 4: adjusted for age, smoking, DBP, APTT, baseline NIHSS score, hematoma volume and early withdrawal of life-sustaining therapy.

natural characteristics of sICH. Second, only admission serum phosphate level was calculated, and therefore, the dynamic change of serum phosphate level was unclear in patients with sICH. Finally, patients with massive ICH who needed surgery were not included, and this may lead to the loss of a large proportion of patients, and the mortality was relatively lower.

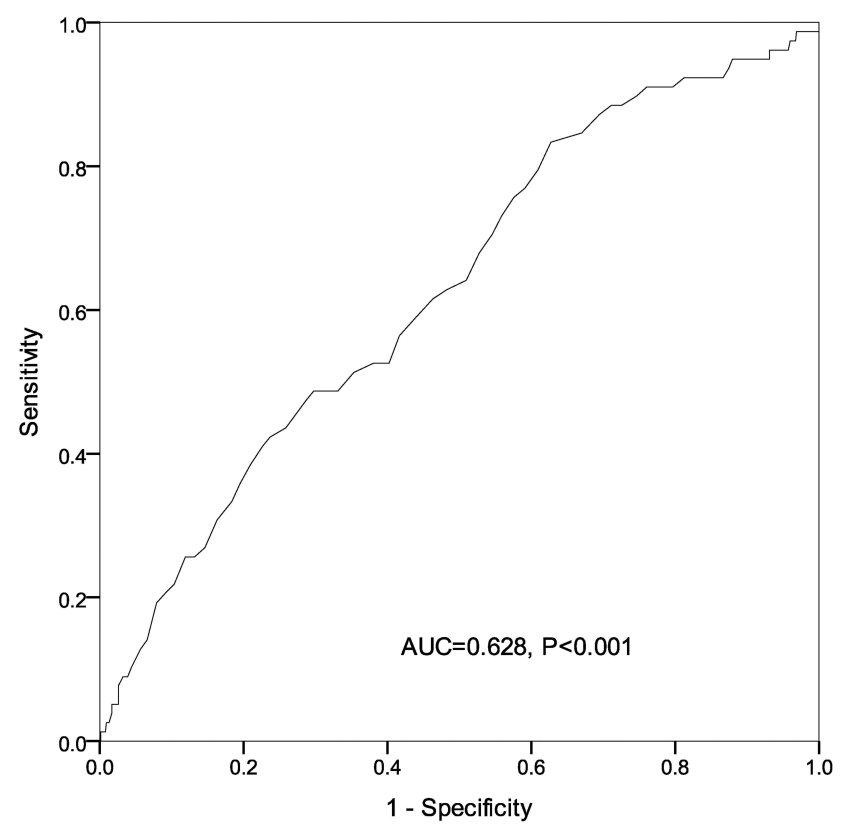

Figure 2 ROC analysis reveals the predictive value of the admission serum phosphate level for all-cause mortality within 90 days among patients with spontaneous intracerebral hemorrhage.
In summary, the results of the present study demonstrate that a low admission serum phosphate is strongly associated with a high risk of mortality in patients with mild-moderate sICH, and hypophosphatemia may be a prognostic marker for all-cause mortality in patients with mild-moderate sICH. Further studies to unravel the underlying mechanisms seem justified.

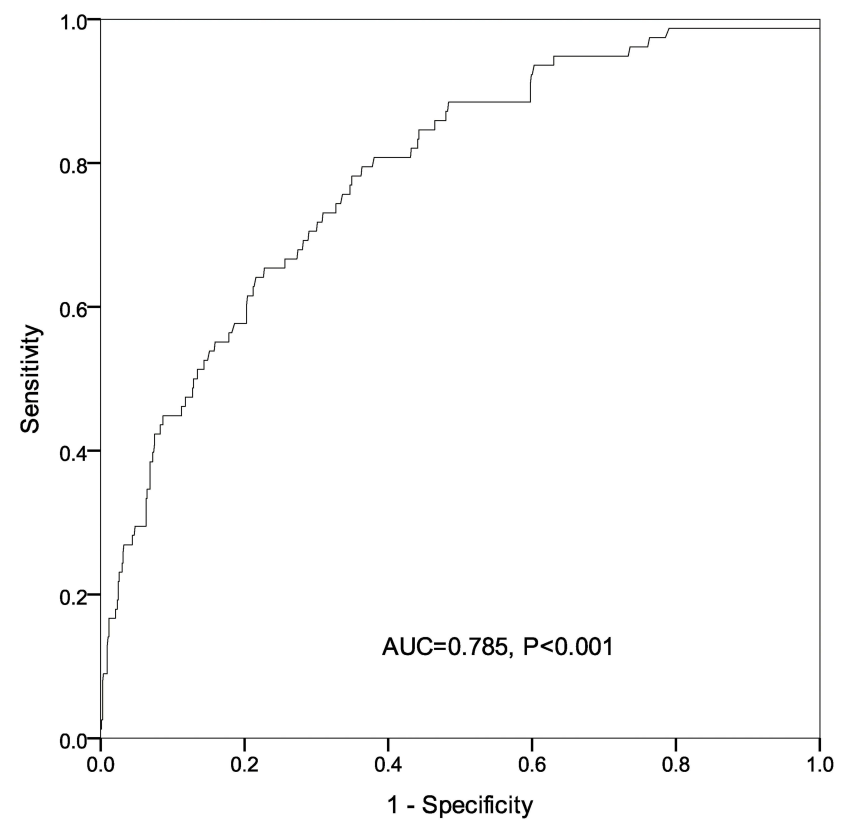

Figure 3 ROC analysis shows the predictive value of admission serum phosphate, hematoma expansion, presence of intraventricular hemorrhage and intracerebral hemorrhage volume on admission for all-cause mortality within 90 days among patients with spontaneous intracerebral hemorrhage. 


\section{Acknowledgments}

We would like to thank all patients participating in this study, and the kind assistance from the Department of Neurology, Yixing People's Hospital and Taicang First People's Hospital during the data collection. This study was supported by the National Natural Science Foundation of China (grant no. 81701302) and the Top Talent Support Program for Young and Middle-aged People of Wuxi Health Committee (grant no. BJ2020108).

\section{Disclosure}

The authors declare no conflicts of interest.

\section{References}

1. Alerhand S, Lay C. Spontaneous intracerebral hemorrhage. Emerg Med Clin North Am. 2017;35(4):825-845. doi:10.1016/j.emc.2017.07.002

2. Josephson CB, Frantzias J, Samarasekera N, Al-Shahi Salman R. The persisting burden of intracerebral haemorrhage: can effective treatments be found? PLoS Med. 2010;7(10):e1000353. doi:10.1371/journal.pmed.1000353

3. Fewel ME, Thompson BG Jr, Hoff JT. Spontaneous intracerebral hemorrhage: a review. Neurosurg Focus. 2003;15(4):E1. doi:10.31 71/foc. 2003.15.4.0

4. Al-Shahi Salman R, Labovitz DL, Stapf C. Spontaneous intracerebral haemorrhage. BMJ. 2009;339:b2586. doi:10.1136/bmj.b2586

5. Bai Q, Sheng Z, Liu Y, Zhang R, Yong VW, Xue M. Intracerebral haemorrhage: from clinical settings to animal models. Stroke Vasc Neurol. 2020;5(4):388-395.

6. Rincon F, Mayer SA. Intracerebral hemorrhage: getting ready for effective treatments. Curr Opin Neurol. 2010;23(1):59-64. doi:10.1097/WCO.0b013e3283352c01

7. Hostettler IC, Seiffge DJ, Werring DJ. Intracerebral hemorrhage: an update on diagnosis and treatment. Expert Rev Neurother. 2019;19 (7):679-694. doi:10.1080/14737175.2019.1623671

8. Veltkamp R, Purrucker J. Management of spontaneous intracerebral hemorrhage. Curr Neurol Neurosci Rep. 2017;17(10):80. doi:10.100 7/s11910-017-0783-5

9. Moulin S, Cordonnier C. Prognosis and outcome of intracerebral haemorrhage. Front Neurol Neurosci. 2015;37:182-192.

10. Gutiérrez OM. Increased serum phosphate and adverse clinical outcomes: unraveling mechanisms of disease. Curr Opin Nephrol Hypertens. 2011;20(3):224-228. doi:10.1097/MNH.0b013e 328343 ea 70

11. Lederer E. Why would serum phosphorus correlate with cardiovascular risk, and how is the clinician supposed to use this information? Clin Nephrol. 2017;87(1):1-10. doi:10.5414/CN108916

12. Padelli M, Leven C, Sakka M, Plée-Gautier E, Carré JL. Causes, consequences and treatment of hypophosphatemia: a systematic review. Presse Med. 2017;46(11):987-999. doi:10.1016/j.lpm.2017 .09 .002

13. Park W, Kim BS, Lee JE, et al. Serum phosphate levels and the risk of cardiovascular disease and metabolic syndrome: a double-edged sword. Diabetes Res Clin Pract. 2009;83(1):119-125. doi:10.1016/j. diabres.2008.08.018

14. Demirjian S, Teo BW, Guzman JA, et al. Hypophosphatemia during continuous hemodialysis is associated with prolonged respiratory failure in patients with acute kidney injury. Nephrol Dial Transplant. 2011;26(11):3508-3514. doi:10.1093/ndt/gfr075
15. Otero TMN, Canales C, Yeh DD, et al. Association of serum phosphate levels and anemia in critically ill surgical patients. JPEN J Parenter Enteral Nutr. 2017;41(6):986-992. doi:10.1177/01486 07115626415

16. Yamada S, Tsuruya K, Taniguchi M, et al. Association between serum phosphate levels and stroke risk in patients undergoing hemodialysis: the Q-cohort study. Stroke. 2016;47(9):2189-2196. doi:10.1161/STROKEAHA.116.013195

17. Junttila E, Koskenkari J, Ala-Kokko T. Hypophosphatemia after nontraumatic intracranial hemorrhage. Acta Anaesthesiol Scand. 2017;61(6):641-649. doi:10.1111/aas.12903

18. You S, Han Q, Xu J, et al. Serum calcium and phosphate levels and short- and long-term outcomes in acute intracerebral hemorrhage patients. J Stroke Cerebrovasc Dis. 2016;25(4):914-920. doi:10.1016/j.jstrokecerebrovasdis.2015.12.023

19. Divani AA, Majidi S, Luo X, et al. The ABCs of accurate volumetric measurement of cerebral hematoma. Stroke. 2011;42(6):1569-1574. doi:10.1161/STROKEAHA.110.607861

20. Kwah LK, Diong J. National Institutes of Health Stroke Scale (NIHSS). J Physiother. 2014;60(1):61. doi:10.1016/j.jphys.2013.12.012

21. Torres RB, Giovanni Terzi RG, Eriras Falcão AL, Höehr NF, Dantas Filho VP. Hypophosphatemia in severe traumatic brain injury. Rev Bras Terap Intens. 2005;17:116-120.

22. Faul M, Coronado V. Epidemiology of traumatic brain injury. Handb Clin Neurol. 2015;127:3-13.

23. Polderman K, Bloemers F, Peerdeman S, Girbes A. Hypomagnesemia and hypophosphatemia at admission in patients with severe head injury. Crit Care Med. 2000;28(6):2022-2025. doi:10.1097/ 00003246-200006000-00057

24. Polderman KH, Peerdeman SM, Girbes AR. Hypophosphatemia and hypomagnesemia induced by cooling in patients with severe head injury. $J$ Neurosurg. 2001;94(5):697-705. doi:10.3171/jns.200 1.94.5.0697

25. García Martín A, Varsavsky M, Cortés Berdonces M, et al. Phosphate disorders and clinical management of hypophosphatemia and hyperphosphatemia. Endocrinol Diabetes Nutr. 2020;67(3):205-215. doi:10.1016/j.endinu.2019.06.004

26. Zhang D, Zhuang Z, Wei Y, et al. Association of admission serum glucose-phosphate ratio with severity and prognosis of aneurysmal subarachnoid hemorrhage. World Neurosurg. 2019;127:e1145-e1151. doi:10.1016/j.wneu.2019.04.071

27. Qiu SZ, Zheng GR, Chen B, Huang JJ, Shen J, Mao W. Prognostic value of admission serum glucose-phosphate ratio in predicting the 6-month outcome of patients with severe traumatic brain injury: a retrospective study. Clin Chim Acta. 2020;510:659-664. doi:10.1016/j.cca.2020.08.038

28. Shoji T, Masakane I, Watanabe Y, Iseki K, Tsubakihara Y. Elevated non-high-density lipoprotein cholesterol (non-HDL-C) predicts atherosclerotic cardiovascular events in hemodialysis patients. CJASN. 2011;6(5):1112-1120. doi:10.2215/CJN.09961110

29. Kalantar-Zadeh K, Kuwae N, Regidor DL, et al. Survival predictability of time-varying indicators of bone disease in maintenance hemodialysis patients. Kidney Int. 2006;70(4):771-780. doi:10.1038/sj.ki.5001514

30. Larsen VH, Waldau T, Gravesen H, Siggaard-Andersen O. Erythrocyte 2,3-diphosphoglycerate depletion associated with hypophosphatemia detected by routine arterial blood gas analysis. Scand J Clin Lab Invest Suppl. 1996;224:83-87. doi:10.3109/00365519609088626

31. Ventura-Clapier R, Moulin M, Piquereau J, et al. Mitochondria: a central target for sex differences in pathologies. Clin Sci (Lond). 2017;131(9):803-822. doi:10.1136/svn-2020-000334

32. Johnson MA, Tekkanat K, Schmaltz SP, Fox IH. Adenosine triphosphate turnover in humans. Decreased degradation during relative hyperphosphatemia. J Clin Invest. 1989;84(3):990-995. doi:10.1 172/JCI114263 
33. Haglin L. Using phosphate supplementation to reverse hypophosphatemia and phosphate depletion in neurological disease and disturbance. Nutr Neurosci. 2016;19(5):213-223. doi:10.1179/ 1476830515 Y.0000000024
34. Noda H, Iso H, Irie F, et al. Low-density lipoprotein cholesterol concentrations and death due to intraparenchymal hemorrhage The Ibaraki Prefectural Health Study. Circulation. 2009;119 (16):2136-2145. doi:10.1161/CIRCULATIONAHA.108.795666

\section{Publish your work in this journal}

Risk Management and Healthcare Policy is an international, peerreviewed, open access journal focusing on all aspects of public health, policy, and preventative measures to promote good health and improve morbidity and mortality in the population. The journal welcomes submitted papers covering original research, basic science, clinical \& epidemiological studies, reviews and evaluations, guidelines, expert opinion and commentary, case reports and extended reports. The manuscript management system is completely online and includes a very quick and fair peer-review system, which is all easy to use. Visit http://www.dovepress.com/testimonials.php to read real quotes from published authors. 